Methods Scientific evidence from recently published reviews, including a recent review our team completed, will be synthesised. Evidence from practitioners' expertise and worker experiences are being collected using a web-based survey, focus groups, and interviews with representatives from various stakeholder groups from multiple sectors. We are using the Public Health Agency of Canada's best practices portal to structure data collection of workplace practices and policies. We are synthesising the evidence gathered from stakeholders with that from recently published systematic reviews.

Result Recent systematic review results revealed 61 high and medium studies addressing MSD. The studies described 30 different intervention categories. There was strong evidence that resistance training has a positive effect and moderate evidence that stretching, using a feedback mouse, and workstation forearm supports have positive effects. However the level of evidence was too low to make recommendations for many other interventions. The survey and interview/focus groups to collect practice-based evidence are ongoing.

Discussion The presentation will focus on current policies and practices described by our practitioner and workplace audiences as compared to the scientific evidence. The discussion will outline the synthesis of evidence and co-creation (with OHS stakeholders) of a practical guide to help workplaces develop and implement effective practices and policies to prevent MSD and help workers with MSD return to work safely.

\section{ON - LINE ERGONOMIC WEB ASSESSMENTS - A VALUABLE, COST-EFFECTIVE TOOL FOR PREVENTION OF MUSCULO-SKELETAL DISCOMFORT IN GLOBAL CORPORATE OFFICES}

${ }^{1}$ Kishore Madhwani, ${ }^{2} \mathrm{HV}$ Ravimohan, ${ }^{3}$ Nadarajan Guharaja, ${ }^{4}$ Akhtur Salman, ${ }^{5} \mathrm{VR}$ Mohil, Beliappa2. 'Hindustan Unilever Limited, Mumbai, India; ${ }^{2}$ Hindustan Unilever Limited, Bangalore, India; ${ }^{3}$ Unilever Asia Private Limited, Singapore, Singapore; ${ }^{4}$ Unilever Pakistan Private Limited, Lahore, Pakistan; ${ }^{5}$ Hindustan Unilever Limited, Chhindwara, India

\subsection{6/oemed-2018-ICOHabstracts. 777}

Introduction Globally, it is vital to create office ergonomic awareness amongst corporate employees with emphasis on ergonomic arrangement of workstations, maintaining ideal work postures, optimising chair functions and performing desk stretches.Objective is to promote safer working by exploring cost-effective communication methods to achieve behaviour change.

Methods Invitation was sent to all employees to take up online Nordic questionnaire, a screening tool for musculoskeletal symptoms, shared in local languages on 2 occasions - baseline and after 3 months in Unilever offices at Sri Lanka, Singapore and Malaysia. After completing, employee is guided to animation graphics and trained on correct postures and office ergonomics with animation graphics, $n=203$. Study is currently being undertaken in Pakistan and India (results to be shared subsequently).

Results Approximately 10\% employees had some musculoskeletal symptoms. $85 \%$ of the symptomatic had chronic musculoskeletal symptoms of 1 year duration. However, only $40 \%$ of these employees had ongoing symptoms at the time of evaluation (past 7 days). A subsequent 3 months evaluation after web-based intervention showed a significant $35 \%$ decline in ongoing symptoms. Behavioural interventions responsible for this decline were attributable to:
$70 \%$ employees could arrange their workstation ergonomically, as against $49 \%$ pre intervention.

$83 \%$ employees performing desk stretches as against 59\% pre intervention.

$70 \%$ of the employees taking rest breaks as against $65 \%$ pre intervention.

97\% employees reported animation graphics were educative and motivating.

Discussion The web - based evaluation appears to be an excellent, cost-effective method bringing about desired behaviour change in ideal work-posture maintenance. Hence, newer technology using web based animation graphics is a highly efficient tool to create office ergonomics awareness and has the potential to become a best practice in countries where language is a communication barrier and an on-site visit is not feasible.

\section{THEORETICAL IMPACT OF WORKPLACE-BASED PRIMARY PREVENTION OF CARPAL TUNNEL SYNDROME IN A FRENCH REGION: A PILOT STUDY}

\begin{abstract}
${ }^{1} Y$ Roquelaure, ${ }^{1} \mathrm{~A}$ Petit, ${ }^{2} \mathrm{E}$ Chazelle, ${ }^{3} \mathrm{~A}$ Descatha, ${ }^{4} \mathrm{~B}$ Evanoff, ${ }^{1} \mathrm{f}$ Bodin, ${ }^{1,2} \mathrm{~N}$ Fouquet. ${ }^{1}$ INSERM, U1085, IRSET, ESTER Team, University of Angers, Angers, France; ${ }^{2}$ French national public health agency, Direction of Occupational Health, Saint-Maurice, France; ${ }^{3}$ INSERM UMS 011, University Versailles St-Quentin, Versailles, France; ${ }^{4}$ Washington University School of Medicine, Division of General Medical Sciences, St. Louis, USA
\end{abstract}

\subsection{6/oemed-2018-ICOHabstracts. 778}

Introduction To assess the theoretical impact of workplacebased primary interventions designed to reduce exposure to personal and/or work-related risk factors for carpal tunnel syndrome (CTS).

Methods Cases of CTS were assessed using regional hospital discharge records for persons aged 20-59 in 2004. Using work-related attributable fractions (AFEs), we estimated the number of work-related cases of CTS (WR-CTS) in high-risk jobs. We simulated three theoretical scenarios of workplacebased primary prevention for jobs at risk: a mono-component work-centred intervention reducing the incidence of WR-CTS by $10 \%(10 \%-\mathrm{WI})$, and multicomponent global interventions reducing the incidence of all surgical cases of CTS by $5 \%$ and $10 \%$ by targeting personal and work risk factors.

Result Nine jobs at high risk of CTS were identified in the region, amounting to $1,618[1,143-2,233]$ CTS cases, of which 952 [488-1,575] were WR-CTS. The 10\%-WI, 5\% GI and $10 \%$ GI hypothetically prevented 95 [49-158], 81 [57112] and 162 [114-223] CTS cases, respectively. The hypothetical preventive efficiency varied depending on the job. The $10 \%$ GI had the greatest impact regardless of the job. The impact of the $10 \%$-WI interventions was high only in jobs at highest risk and AFEs (e.g. food industry jobs). The 10\%-WI and 5\% GI had a similar impact for moderate-risk jobs (e.g. healthcare jobs).

Discussion This study found that work-related CTS cases were concentrated in several high-risk jobs. Simulated workplacebased WI and GI showed that preventive efficiency varied depending on the intervention design, the number of workers in different jobs and the proportion of work-related CTS. This suggests that prevention efforts to reduce exposure to work-related risk factors should focus on high-risk jobs. Reducing CTS rates will also require strategies to reduce personal risk factors, particularly in jobs with low levels of workrelated risk of CTS. 